\title{
Antimicrobial Resistance Profile of Helicobacter pylori Clinical Isolates in Duhok City, Kurdistan Region/Iraq
}

\author{
Ali Yahya Saeed ${ }^{1}$, OdeeshYohana Odeesh ${ }^{2}$, KhoshiSalim \\ Abdulhafith ${ }^{3}$,NawfalRasheed Hussein ${ }^{4}$ \\ ${ }^{1}$ Department of Biology, Faculty of Science, University of Duhok, Kurdistan Region/ Iraq \\ ${ }^{2}$ Gastroenterology and hepatology center, Azadi Teaching Hospital, Directorate General Health-Duhok, \\ Ministry of Health, Kurdistan Region, Iraq \\ ${ }^{3}$ Azadi Teaching Hospital Laboratory, Directorate General Health-Duhok, Ministry of Health, Kurdistan \\ Region, Iraq. \\ ${ }^{4}$ Department of Internal Medicine, Faculty of Medical Sciences, University of Duhok, Kurdistan Region, Iraq
}

\begin{abstract}
Studies have been referred to the increasing the numbers of $H$. pylori isolates against antibiotics in the world and account for recurrent and unresponsiveness of many cases of infections. In Iraq, particularly Kurdistan region there are no data regarding the antibiotic resistance profile of $H$. pylori isolated from clinical cases.The purpose of this work was to investigate the antimicrobial resistance profile of $H$. pylori isolates from clinical cases against clarithromycin, levofloxacin, amoxicillin, metronidazole, ciprofloxacin and tetracycline. In this study 150 antral biopsies were sampled from patients referred to the Duhokhepatology and gastroenterology center, Azadi Teaching Hospital, Duhok, Kurdistan Region, Iraq for endoscopy examination between March and May, 2014. H. pylori was isolated from 30 (20\%) out of 150 patients and their antimicrobial susceptibility were checked by disc diffusion assay.

All isolates were susceptible to levofloxacin but with variable resistance patterns to metronidazole, tetracycline, ciprofloxacin, clarithromycin and amoxicillin which were $53.3 \%, 16.6 \%, 13.4 \%, 10 \%$ and $6.7 \%$ respectively. The patterns of resistance were differed from resistance to 4 combined antimicrobial agents to 2 combined antimicrobial agents.

Conclusions: A high percentage of resistance to metronidazole was detected and it should be considered when prescribing drugs to the patients by the clinicians. Periodic evaluation the antimicrobial resistance profile of $H$. pylori clinical isolates against commonly prescribed drugs in order to prevent generation of resistant strains and to choose the most effective therapeutic strategy in the area.
\end{abstract}

\section{Introduction}

Helicobacter pylori (H. pylori) is the only known pathogen that inhabits the stomach of about half people throughout the world and classified as a class 1 carcinogen [1,2]. This bacterium can remain asymptomatic or can cause gastritis, gastric and duodenal ulcer and mucosa-associated lymphoid tissue lymphoma [3]. H. pylori is widespread in Kurdistan region of in which $65 \%$ of dyspeptic patients were positive for $\mathrm{H}$. pylori by culture [4] and $70.93 \%$ by ELISA IgG [5]. Therefore, it has a significant health problem which requires eradication by using antimicrobial drugs [6,7]. Studies had been referred to the important role of antimicrobial drugs in the controlling of $H$. pylori infection. Although $H$. pylori exhibits susceptibility to most antibiotics in vitro, but few antibiotics can be used successfully for eradication of $H$. pylori, i.e. amoxicillin, clarithromycin, metronidazole and tetracycline.Successful treatment of $H$. pylori requires combination of two antibiotics plus proton pump inhibitors [8]. One problem associated with widespread use of antibiotics against $H$. pylori is the generation of resistant strains throughout the world which varies with geographical region $[9,10]$. Because no data are available on the antimicrobial resistance of $H$. pylori infection in our area with increasing the numbers of unresponsiveness cases to the anti- $\mathrm{H}$. pylori antimicrobial drugs, therefore the aim of the present work to study the antimicrobial susceptibility of $\mathrm{H}$. pylori strains isolated from clinical cases and to find out the most effective drugs against $H$. pylori infection.

\section{Materials and Methods}

2.1. Patients

A total of 150 patients referred to the Duhokhepatology and gastroenterology center, Azadi Teaching hospital, Duhok, Kurdistan Region, Iraq for endoscopy examination between March and May, 2014 were enrolled in the study. Men were 68 with ages from 18-85 years old (average age: 51) and 82 were women with ages from 2-90 years old (average age: 46). Only Patients without receiving any antibiotics for previous two weeks were enrolled in the study. Antral biopsy was obtained from each patient and placed in sterile tubes containing thioglycolate broth (Himidia. India) and immediately sent to the microbiology laboratory, Faculty of Science for 
processing.

2.2. Isolation and Identification of $H$. pylori

Biopsies were dispersed under aseptic conditions and streaked on the surface of Columbia blood agar (Oxoid, UK) enriched with 5\% sheep blood and supplemented with Dent supplement (Bio Merieux, France). All cultured plates were incubated at $37{ }^{\circ} \mathrm{C}$ for one week in microaerobic conditions $\left(5 \% \mathrm{O}_{2}, 15 \% \mathrm{CO}_{2}, 85 \% \mathrm{~N}_{2}\right)$ using Campy gas packs (Bio Merieux, France). Identification was based on the colony morphology, Gram staining and enzymatic reactions such as catalase, oxidase and urease tests. The isolates were sub-cultured on Columbia blood agar and incubated at $37{ }^{\circ} \mathrm{C}$ for 3 days under microaerobic conditions to obtain purified bacterial growth.

\subsection{Antibiotic Susceptibility Test}

All isolates were tested against six different antimicrobial drugs using disc diffusion assay, based on the method followed by Lopez Brea and Alarcon [11]. Bacterial suspensions were prepared in $1.0 \mathrm{ml}$ of sterile saline solution. $0.5 \mathrm{ml}$ of suspensions was spread on Mueller-Hinton agar plates (BioLab. UK) supplemented with 5\% sheep blood and allowed to dry for 10 minutes. Six different discs were tested for each plate using levofloxacin $(5 \mu \mathrm{g})$, clarithromycin $(5 \mu \mathrm{g})$, metronidazole $(30 \mu \mathrm{g})$, ciprofloxacin $(10 \mu \mathrm{g})$, amoxicillin $(25 \mu \mathrm{g})$ and tetracycline $(10 \mu \mathrm{g})$ (Oxoid, UK). Plates were incubated under microaerobic conditions for 72 hours. The inhibition zones were measured in millimeters $(\mathrm{mm})$ and interpreted as sensitive $(\mathrm{S})$ or resistance $(\mathrm{R})$ in comparison to the National Committee for Clinical Laboratory Standards (NCCLS) [12].

\section{Results}

From 150 antral biopsies, 30 (20\%) strains of $H$. pylori were, in which 16 strains were from males and 14 strains were from females. The resistance profile of six different antibiotics was checked by disc diffusion test as shown in Fig.1.

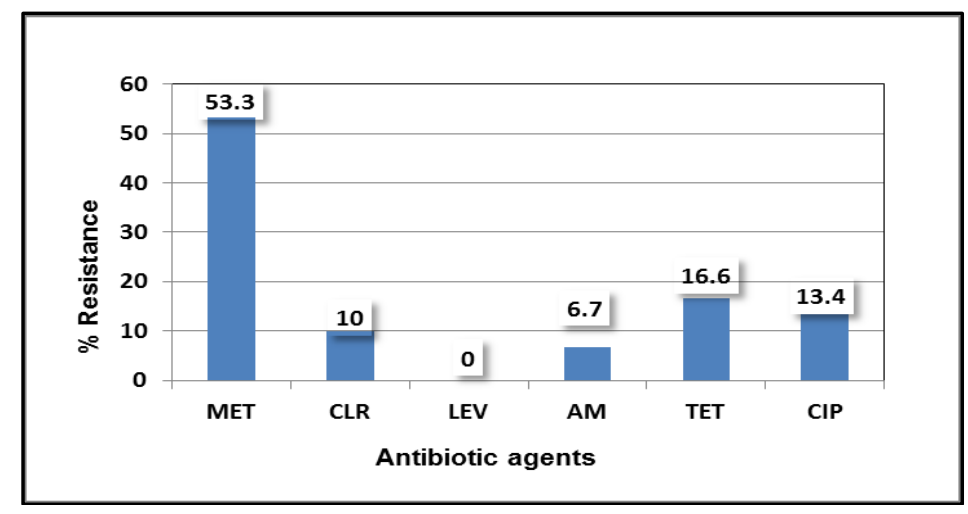

Figure 1. Antimicrobial resistance profile of $H$. pylori clinical isolates.

The highest percentage of resistance was 53.3\% (16/30) with metronidazole and the lowest was $0.0 \%$ with levofloxacin, while for tetracycline, ciprofloxacin, clarithromycin and amoxicillin was $16.6 \%(5 / 30), 13.4 \%$ $(4 / 30), 10(3 / 30)$ and $6.7 \%(2 / 30)$ respectively (Table 1$)$.

Table 1: Number and percentages of $H$. pylori strains resistant to antibiotics

\begin{tabular}{|l|l|}
\hline Resistance No.(\%) & Antibiotics \\
\hline $16(53.3)$ & Metronidazole (MET) \\
\hline $5(16.6)$ & Tetracycline (TET) \\
\hline $4(13.4)$ & Ciprofloxacin (CIP) \\
\hline $3(10.0)$ & Clarithromycin (CLR) \\
\hline $2(6.7)$ & Amoxicillin (AM) \\
\hline $0(0.0)$ & Levofloxacin (LEV) \\
\hline
\end{tabular}

Resistant strains of $H$. pylori were found against 5 different combined antimicrobial agents and the patterns of resistance were different from resistance to 4 combined antimicrobial agents to 2 combined antimicrobial agents (Table 2). 
Table 2: Number of resistant $H$. pylori isolates to combine antimicrobial agents

\begin{tabular}{|l|l|}
\hline Resistance No. $(\%)$ & Antimicrobial agents \\
\hline $1(3.3)$ & MET+TET+CIP+AM \\
\hline $1(3.3)$ & MET+TET+CIP \\
\hline $3(10)$ & MET+TET \\
\hline $1(3.3)$ & MET+CIP \\
\hline $2(6.7)$ & MET+AM \\
\hline
\end{tabular}

Abbreviations: MET, Metronidazole; TET, Tetracycline; CIP, Ciprofloxacin; AM, Amoxicillin.

\section{Discussion}

H. pylori becomes widely distributed throughout the world including Kurdistan region of Iraq in which studies had found that $65 \%$ of the dyspeptic patients were positive for $H$. pylori by culture [4] and $70.93 \%$ seropositive by ELISA IgG [5]. Antimicrobial drugs are extensively and empirically used for the treatment of $H$. pylori infections using different antimicrobial drugs. Furthermore, self-medication is widely practiced in the area because people can obtain antibiotics from pharmacies without physician prescriptions which lead to the propagation of drug resistant strains of $H$. pylori in the area as most of the clinicians complaining from increasing the number of recurrent or even non-responsiveness cases of $H$. pylori associated peptic ulcer.

There is no data on antimicrobial resistance profile of $H$. pylori in the area; therefore the study evaluated the resistant profile of $30 \mathrm{H}$. pylori isolates isolated from clinical cases. All isolates showed varied patterns of resistance against metronidazole, tetracycline, ciprofloxacin, clarithromycin and amoxicillin which were 53.3\%, $16.6 \%, 13.4 \%, 10 \%$ and $6.7 \%$ respectively, while all isolates were sensitive to levofloxacin. Resistance to drug combination was found among studied isolates in which $3.3 \%$ of isolates were resistant to four combined drugs, $3.3 \%$ to three combined drugs and variable resistant patterns to two combined drugs (Table 2). High rate of resistance $(51.3 \%)$ was found to metronidazole which was comparable to that recorded by [13, 14, 15] who found that $57.5 \%, 51.5 \%$ and $56.3 \%$ of $\mathrm{H}$. pylori isolates from Iranian patients were resistance to metronidazole respectively and Mansour et al., [16] found that 56.8\% of the H. pylori isolates from Tunisian patients were resistance to metronidazole, but much lower than those found by Aboderin et al., [17] who found that $100 \%$ of H. pylori isolates from Nigerian patients were resistance to metronidazole and Majlesi et al.,[18] who found $63.8 \%$ of $\mathrm{H}$. pylori isolates from Iran were resistance to metronidazole. This high rate of resistance to metronidazole may be due to the wide use of this drug for other infections such as dental, gynecological and parasitic infections $[19,20]$. Resistance to clarithromycin was $10 \%$ which is lower than those recorded in other part of the world such as Mohammedi et al., [13], Mirzaei et al., [15] and Majlesi et al., [18] who found that $26.5 \%, 16.7 \%$ and $14.6 \%$ of the $H$. pylori isolates from Iranian patients were resistance to clarithromycin respectively, Mansour et al, (2010) from Tunisia found 15.4\% were resistance and Aboderin et al., [17] from Nigeria found $100 \%$ were resistance. Resistance to clarithromycin can be developed either by using this drug as a key component of the drug combination used against $H$. pylori or used for the treatment of respiratory tract infection or due to the previous consumption of macrolides which induce cross resistance to clarithromycin [21]. Resistance to fluoroquinolones ciprofloxacin and levofloxacin was different in which $13.4 \%$ of the isolates were resistance to ciprofloxacin and while all isolates were sensitive to levofloxacin. Ciprofloxacin is widely used in our area against various infections, while levofloxacin is a new drug; therefore resistance to ciprofloxacin is predictable. Among $\beta$-lactam antibiotics, amoxicillin is the only prescribed and commonly included in the therapeutic protocols for the treatment of $H$. pylori infection [22]. Among 30 studied isolates $2(6.7 \%)$ were resistant to amoxicillin which was lower in comparison to that detected in Nigeria by Aboderin et al., [17] who found that $100 \%$ were resistance, while higher than those recorded by Mohammedi et al., [13] and Mirzaei et al., [15] who found that $1.6 \%$ and $4.2 \%$ of the H. pylori isolates from Iranian patients were resistance to amoxicillin respectively. Amoxicillin still can be used against $H$. pylori infection when combined with other drugs. Resistance to tetracycline was $16.6 \%$ which was higher than those recorded by Majlesi et al., [18] who found that $2.4 \%$ of the isolates from Iran were resistance to tetracycline, while another studies from Iran did not find H. pylori isolates resistance to tetracycline $[13,14]$. This discrepancies in the results can be attributed to many factors such methodology, geographical variation and treatment regimens.

\section{Conclusions}

Drug resistance strains of $H$. pylori are found against different antimicrobial agents used for the treatment of $H$. pylori infection and periodic monitoring of antibiotic resistance profile is necessary for physicians to choose the effective therapeutic strategy. Our results suggest that metronidazole should not be included in the treatment regimens used for $\mathrm{H}$. pylori in our region. 


\section{References}

[1]. M. Sirous, J.F. Mehrabadi, N.E. Daryani, S. Eshraghi, S. Hajikhani, M.H. Shirazi, Prevalence of antimicrobial resistance in Helicobacter pylori isolates from Iran. Afr J Biotech, 9 (36), 2011, 5962-5965.

[2]. Fallahi G.H. Fallahi, S. maleknejad, Helicobacter pylori culture and antimicrobial resistance in Iran. Indian J Pediatrics, 74 (2), 2007, 127-130.

[3]. Ernst P.B. Ernst, B.D. Gold, The disease spectrum of Helicobacter pylori: the immunopathogenesis of gastroduodenal ulcer and gastric cancer. Annu Rev Microbiol, 54, 2000,615-640.

[4]. A.Y. Saeed and S.M. Pedawi, Prevalence of Helicobacter pylori among dyspeptic patients. Journal of ZankoySulaimani, 5(2), 2002, 37-42.

[5]. H.M.S. Saadi, Evaluation of ELISA IgG,IgM and IgA for the diagnosis of Helicobacter pylori. 2014. M.Sc. Thesis. University of Duhok, Kurdistan Region of Iraq.

[6]. S.H. Ghasemian, H. Tavakkoli, A. Mojtahedi, R. Salehei , B. Soleimani, E. Pishva, Correlation of CagA positive Helicobacter pylori Infection with clinical outcomes in Al-Zahra hospital, Isfahan, Iran. J Res Med Sci, 13(4), 2008,196-201.

[7]. K. Dzierzanowska-Fangrat, E. Rozynek, D. Celinska-Cedro, M. Jarosz, J. Pawlowska, A. Szadkowski, Antimicrobial resistance of Helicobacter pylori in Poland: a multicentre study. Int J Antimicrob Agents, 26(3), 2005, 230-4.

[8]. M.A. Marie, Patterns of Helicobacter pylori resistance to metronidazole, clarithromycin and amoxicillin in Saudi Arabia. J BacteriolVirol, 38(4), 2008,173-178.

[9]. T.F. Haghi, M.A. Mohabbati, M. Amini, D. Hosseini, B.A. Talebi, Helicobacter pylori Resistance to Metronidazole and Clarithromycin in Dyspeptic Patients in Iran. Iran Red Cres Med J, 12(4), 2010, 409-412.

[10]. C.T. Hu, P.Y. Chiou, C.C. Wu, Y.H. Tseng, Y.J. Chang, N.T. Lin, Analysis of Resistance to Clarithromycin and Virulence Marker s in Helicobacter pylori Clinical Isolates from Eastern Taiwan. Tzu Chi Med J, 21(2), 2009,123-128.

[11]. M. Lopez Brea, T. Alarcon: Sensibilidadantimicrobiana en lainfeccionpor Helicobacter pylori. En: Lopez Brea M. Helicobacter pylori microbiologia, clinicaytratamiento; Madrib; Morsby/DoymaLibros. 1995; 32-53.

[12]. National Committee for Clinical Laboratory Standards. Approved Standard M7-A5: Methods/or Dilution Antimicrobial Susceptibility Tests/or Bacteria That Grow Aerobically (National Committee for Clinical laboratory Standards, Wayne, PA) 2000.

[13]. M.Mohammedi, D. Doroud, N. Mohajerani, S. Massarrat, Helicobacter pylori antibiotic resistance in Iran. World J Gastroenterol, 11(38),2005, 6009-13.

[14]. M. Sirous, J.F.Mehrabadi, N.E. Daryani, S. Eshraghi, S. Hajikhani, M.H. Shirazi, Prevalence of antimicrobial resistance in Helicobacter pylori isolates from Iran. African J Biotech, 9(36), 2010, 5962-5.

[15]. N. Mirzaei, F. Poursina, J. Faghri, M. Talebi, M.R. Khataminezhad, A. Hasanzadeh , H.G. Safaei, Prevalence of Resistance of Helicobacter pylori Strains to Selected Antibiotics in Isfahan, Iran. Jundishapur Journal of Microbiology, 6 (5), 2103, 1-6.

[16]. B. Mansour, Ch. Burucoa , M. Zribi, A. Masmoudi, S. Karoui, L. Kallel, Primary resistance to clarithromycin, metronidazole and amoxicillin of Helicobacter pylori isolated from Tunisian patients with peptic ulcers and gastritis: a prospective multicentre study. Ann ClinMicrobiolAntimicrob, 9, 2010, 22-22.

[17]. O.A. Aboderin, A.R.Abdu, W.B.Odetoyin, R.N. Okeke, O.O.Lawal, D.A. Ndububa, A.E. Agbakwuru , A. Lamikanra, Antibiotic resistance of Helicobacter pylori from patients in Ile-Ife, South-west, Nigeria. African Health Science, 7(3), 2007,143-147.

[18]. A. Majlesi, S.M. Khprasani, A.R. Khalilian, M.M. Aslani, M. Jaefari, M.Y. Alikhani, Antibiotic susceptibility of Helicobacter pylori clinical isolates in Hamadan, West of Iran. Int J Enterpathog, 01(01), 2013, 8-11. DOI: 10.5812/ijep.9344

[19]. R. Khoshei, H. Shojaei, P. Adibi, A. Shavakhi, M.M. Aslani, N.Daei, Genetic diversity and drug resistance of Helicobacter pylori strains in Isfahan, Iran. Iran J Basic Med Sci, 11(3),2008,174-182.

[20]. Y. Glupczynski, F. Magraud, M. Lopez-Brea, Anderson L.P.Anderson, European multicentre survey of in vitro antimicrobial resistance in Helicobacter pylori. Euro J ClinMirobiol Infect Dis, 11,2000,820-823.

[21]. K.Wolle, P. Malfertheiner, Treatment of Helicobacter pylori. Best Pract. Res. Clin. Gastroenterol, 21,2007, 315-324.

[22]. J. Kohanteb, A. Bazargani, M. Saberi-Firoozi, A. Mobasser, Antimicrobial susceptibility testing of Helicobacter pylori to selected agents by agar dilution method in Shiraz-Iran. Indian J Med Microbiol, 25(4), 2007,374-7. 